\title{
ACCURACY ANALYSIS OF GNSS (GPS, GLONASS AND BEIDOU) OBSEVATION FOR POSITIONING
}

\author{
Khomsin $^{1, *}$, Ira Mutiara Anjasmara ${ }^{1}$, Danar Guruh Pratomo $^{1}$, and Wahyu Ristanto ${ }^{1}$ \\ ${ }^{1}$ Geomatic Engineering Department, ITS Surabaya, Indonesia
}

\begin{abstract}
Global Navigation Satellite System called GNSS is a term used for the entire global navigation that already operate or are in the planning for the future. Some of the satellite that can be used are GPS (Global Positioning System) operated by USA, GLONASS (Global Navigation Satellite System) operated by Rusia and BeiDou/Compass operated by China. Many errors and biases that occur when measuring with GNSS in the field. Theoritically, there are some errors and biases that can be eliminated or subtracted by strength of satellite geometric. One factor to get a good satellite geometric is to increase the number of satellites received by receiver. In general, the more number of satellites received, the better the geometric satellites received by receivers. The development of receiver technology is currently able to capture GPS, GLONASS and BeiDou signals at one time. Thus the receiver can receive many satellites and finally the shape of geometric satellite becomes better. HiTarget V30 is one of the latest GNSS technology on the market today. This receiver is capable of receiving GPS signals, GLONASS and BeiDou at one time of observation. This research will compare the accuracy of positioning using GPS, GLONASS and BeiDou satellite.
\end{abstract}

\section{Introduction}

Recently, the technology of satellite navigation is continuously increasing in number of new applications. A few decades ago, many applications that were just a dream are becoming reality today and the potential applications coverages has grown significantly over the last few years. The unprecedented social, economic, technological and environmental benefits to be gained through these applications are becoming very clear [1].

Global Navigation Satellite System called GNSS is a term used for the entire global navigation satellite system that already operate or are in the planning [2]. Some of which are GPS, GLONASS, Galileo, BeiDou, IRNSS, dan QZSS satellite system. The use of GNSS make the extraterrestrial survey easier and will give better precision. GNSS can not only be used world-wide, all weather and all times but also can be used to determine precise position to all manner of user especially for surveying and geodetic applications [3].

A GNSS receiver calculates position based on data received from satellites. However, there are many sources of errors that, if left uncorrected, cause the position calculation to be inaccurate. Some of these errors, such as those caused by the refraction of the satellite signal as it passes through the ionosphere and troposphere, multipath, cycle slip, orbit, satellite clock and receiver noise [4]. The accuracy of positioning from a GNSS survey will generally depend on 4 (four) factors, namely: the accuracy of the data used, the observation geometry, the observation strategy used, and the data processing strategy. The accuracy of the data used will basically depend on three factors, namely: data type (pseudo range or phase), the quality of the receiver, and the level of error and bias affecting the observation data [5].

The observation geometry includes the observer geometry (network) and satellite geometry that depends on the number of satellites, locations, and distribution of satellites received. Theoretically, the more satellites received, the better satellite geometry [2][6]. The evolving receiver technology can overcome the error of less satellite geometry when retrieving data.

According to [7], GNSS signal combination which is between BeiDou signals (B1 and B2) and GPS signals (L1 and L2) has produced $\mathrm{x}, \mathrm{y}$, and $\mathrm{z}$ coordinates with better accuracy and precision. The combination of GPS signals and GLONASS signals [6] can increase accuracy up to millimeter order for short baselines and order centimeters for long baselines. In this research we will analyze the use of three combinations of GPS, GLONASS and BeiDou satellites using Hi-Target V30 receiver. With the use of more satellites it will get more data which expected to increase the accuracy of GNSS surveys.

*Corresponding author: khomsin95@yahoo.com 


\section{Data and Method}

\subsection{Data}

Locating a suitable area for conducting this research is Surabaya City, East Java. The survey location and the design of baseline survey can be seen in Figure 1 below.

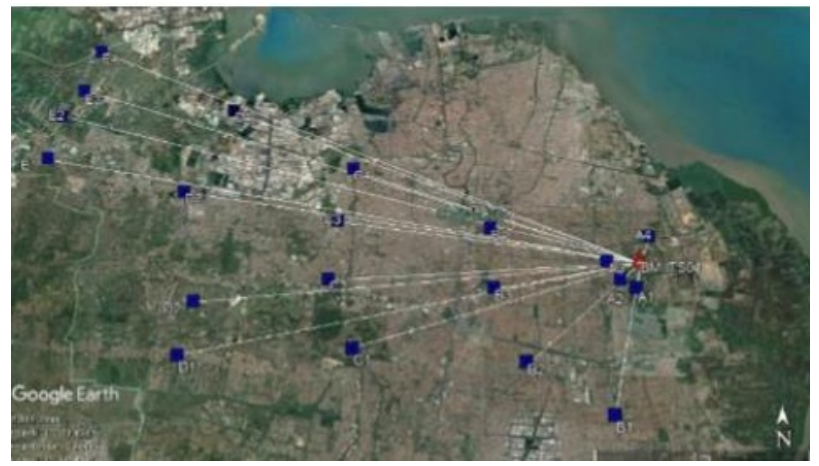

Figure 1. Survey Location and baseline survey plan

\subsection{Instruments}

The study used GNSS receiver Hi-Target V30 to observe the baseline from base to other points (rovers). The instrument specifications and visualizations can be seen in Table 1 and Figure 2.

Table 1. GNSS receiver Hi-Target V30 Specification [8]

\begin{tabular}{l|l}
\hline \multirow{2}{*}{ Measurement } & 220 channels \\
\hline \multirow{3}{*}{$\begin{array}{l}\text { Satellite signal } \\
\text { tracked } \\
\text { simultaneously }\end{array}$} & GPS (L1C/A, L2C, L2E, L5) \\
\cline { 2 - 2 } & BeiDou (B1 and B2) \\
\hline \multirow{3}{*}{$\begin{array}{l}\text { Static and Fast } \\
\text { Static }\end{array}$} & H: $2.5 \mathrm{~mm} \times 0.5 \mathrm{ppm} \mathrm{D}$ \\
\cline { 2 - 2 } & $\mathrm{V}: 5.0 \mathrm{~mm} \times 0.5 \mathrm{ppm} \mathrm{D}$ \\
\hline & H: $8.0 \mathrm{~mm} \times 1.0 \mathrm{ppm} \mathrm{D}$ \\
\cline { 2 - 2 } RTK Surveying & $\mathrm{V}: 15.0 \mathrm{~mm} \times 1.0 \mathrm{ppm} \mathrm{D}$ \\
\hline
\end{tabular}

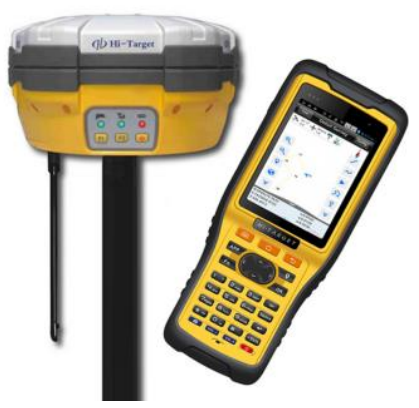

Figure 2. GNSS receiver Hi-Target V30

\subsection{Method}

The first step taken in this research is planning a survey that includes the baseline design and field orientation. In this case, we plan some baselines with distance of $1 \mathrm{~km}$, $5 \mathrm{~km}, 10 \mathrm{~km} 15 \mathrm{~km}$ and $20 \mathrm{~km}$ from a base station. In addition, each baseline distance categories have four baselines (points), so that total observations in this case are twenty baselines. After that is the field orientation to check the location of the points to be measured whether the location meets the requirements or not by looking at multipath and other obstacles.

The next step is the GNSS survey was carried out in accordance with the designs previously made using two V30 Hi-Target GNSS Receivers where one receiver as a base and another as a rover. The observation method used is static with an hour observation and only radial observation with BM ITS-01 as a base and other points as rovers.

After the all of observations are complete, the observation data are downloaded and then to be processed with 6 strategies:

- $1^{\text {st }}$ strategy is GPS, GLONASS and BeiDou signals are processed simultaneously

- $\quad 2^{\text {nd }}$ strategy is both of GPS and GLONASS signal are processed simultaneously

- $\quad 3^{\text {rd }}$ strategy is both of GPS and BeiDou signals are processed simultaneously

- $\quad 4^{\text {th }}$ strategy is both of GLONASS and BeiDou signals are processed simultaneously

- $5^{\text {th }}$ strategy is only GPS signal is processed

- $6^{\text {th }}$ strategy is only GLONASS satellite signal is processed

We could not process only BeiDou signal because the GNSS receiver Hi-Target V30 just received two satellite of BeiDou, namely B1 and B2. Finally, this research will compare coordinate results between one strategy and other strategies. 


\section{Result and Discussion}

\subsection{BM Referenced}

The GNSS observation in this case was carried out by static positioning and radial methods which were tied at the BM ITS - 01 as referenced point. This BM is located in front of the ITS Rectorat building in Sukolilo Campus - Eastern of Surabaya (Figure 3). The coordinates of BM ITS-01 can be seen at Table 2 .

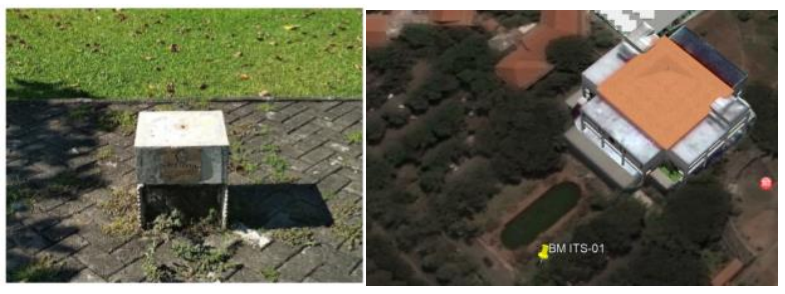

Figure 3. BM ITS - 01 and its location

Table 2. BM ITS-01 coordinate as referenced point

\begin{tabular}{l|l}
\hline \multicolumn{2}{c}{ Coordinate } \\
\hline Easting (m) & 698.076 .449 \\
\hline Northing (m) & 9194686.012 \\
\hline Latitude (S) & $7^{\circ} 16^{\prime} 55.123^{\prime \prime}$ \\
\hline Longitude (E) & $112^{\circ} 47^{\prime} 38.956^{\prime \prime}$ \\
\hline Ellipsoid Height (m) & 36.056 \\
\hline
\end{tabular}

The condition of BM ITS - 01 is relatively still strong and sturdy, and generally the location is free from multipath and other obstacles. Even though there is a little multipath from the surrounding trees, but it can still be reduced by $15^{0}$ mask angle observation.

\subsection{Length of baseline observation}

In this research, the GNSS observation is divided to five categories of length of baselines: $1 \mathrm{~km}(\mathrm{~A}), 5 \mathrm{~km}(\mathrm{~B}), 10$ $\mathrm{km}(\mathrm{C}), 15 \mathrm{~km}$ (D) and $20 \mathrm{~km}$ (E) from the base station. Each category has four measured points, so that a total of twenty points are measured (Table 3 ). The Table 3 below shows that the baseline distance at each point. The length of the baseline obtained cannot be fixed with the planning carried out, because only estimates and location conditions do not make it possible to take measurements so that it must be shifted. However, in general the conditions of GNSS observations are still the same as the planned.

\subsection{Coordinate accuracy analysis of baseline processing}

This GNSS data processing used Hi-Target Geomatics Office (HGO) software to convert format data from HiTarget format to RINEX (Receiver Independence Exchange) format and Topcon Tools 8.2.3 software to perform a baseline (differential positioning) processing. Table 4 to Table 9 shows the accuracy of coordinate results for category $\mathrm{A}, \mathrm{B}, \mathrm{C}, \mathrm{D}$, and $\mathrm{E}$ with $1^{\text {st }}$ strategy to $6^{\text {th }}$ strategy. There are two accuracies, namely horizontal accuracy and vertical accuracy.

Table 3. Length of Baseline observation

\begin{tabular}{|c|c|c|c|}
\hline Base & Category & Point & Distance $(\mathrm{m})$ \\
\hline \multirow{20}{*}{ BM ITS - 01} & \multirow{4}{*}{$1 \mathrm{~km}$} & A1 & 915.584 \\
\hline & & $\mathrm{A} 2$ & 1123.152 \\
\hline & & A3 & 960.171 \\
\hline & & A4 & 968.860 \\
\hline & \multirow{4}{*}{$5 \mathrm{~km}$} & B1 & 5022.564 \\
\hline & & B2 & 4957.415 \\
\hline & & B3 & 6518.977 \\
\hline & & B4 & 5561.453 \\
\hline & \multirow{4}{*}{10 km } & $\mathrm{C} 1$ & 10061.730 \\
\hline & & $\mathrm{C} 2$ & 10096.420 \\
\hline & & $\mathrm{C} 3$ & 10513.090 \\
\hline & & $\mathrm{C} 4$ & 9778.561 \\
\hline & \multirow{4}{*}{15 km } & D1 & 14847.860 \\
\hline & & D2 & 14648.180 \\
\hline & & D3 & 14597.480 \\
\hline & & D4 & 14242.440 \\
\hline & \multirow{4}{*}{$20 \mathrm{~km}$} & E1 & 20628.960 \\
\hline & & E2 & 20492.890 \\
\hline & & E3 & 20848.130 \\
\hline & & E4 & 20860.360 \\
\hline
\end{tabular}

Table 4 shows that the $1^{\text {st }}$ strategy processing using GPS + GLONASS + BeiDou signals together produce horizontal and vertical accuracy less than $1 \mathrm{~cm}$ for length of baseline $\leq 5 \mathrm{~km}$. For the length of baseline from $10 \mathrm{~km}$ to $20 \mathrm{~km}$, this strategy resulted less than $2 \mathrm{~cm}$ for horizontal accuracy and $2.5 \mathrm{~cm}$ for vertical accuracy. These accuracies can be used as ground control point measurements for high resolution satellite image, airborne photogrammetry and complete land registration.

In the $2^{\text {nd }}$ strategy used GPS + GLONASS signals together to process the data, it can be seen in Table 5 that all baselines have the same horizontal and vertical accuracy with the $1^{\text {st }}$ strategy. It means that BeiDou satellite signals namely B1 and B2 has no significant to improve GPS + GLONASS accuracy. Therefore, all the $2^{\text {nd }}$ strategy coordinates accuracies can be used as ground control point and independent check point measurements for high resolution satellite image and airborne Lidar and photogrammetry rectification and orthorectification process. In addition to these accuracies can also be used 
as control points for surveying and mapping and complete land registration project.

Table 4. Coordinate accuracy from GPS + GLONASS + BeiDou processing ( $1^{\text {st }}$ Strategy)

\begin{tabular}{|c|c|c|c|}
\hline Category & Point & $\begin{array}{l}\text { Horizontal } \\
\text { RMS (m) }\end{array}$ & $\begin{array}{l}\text { Vertical } \\
\text { RMS (m) }\end{array}$ \\
\hline \multirow{4}{*}{$1 \mathrm{~km}$} & A1 & 0.2 & 0.5 \\
\hline & $\mathrm{A} 2$ & 0.3 & 0.6 \\
\hline & A3 & 0.5 & 0.8 \\
\hline & A4 & 0.3 & 0.4 \\
\hline \multirow{4}{*}{$5 \mathrm{~km}$} & B1 & 0.3 & 0.5 \\
\hline & B2 & 0.3 & 0.4 \\
\hline & B3 & 0.4 & 0.8 \\
\hline & B4 & 0.3 & 0.5 \\
\hline \multirow{4}{*}{$10 \mathrm{~km}$} & $\mathrm{C} 1$ & 1.1 & 2.0 \\
\hline & $\mathrm{C} 2$ & 1.4 & 2.3 \\
\hline & $\mathrm{C} 3$ & 1.9 & 1.4 \\
\hline & $\mathrm{C} 4$ & 0.7 & 1.2 \\
\hline \multirow{4}{*}{$15 \mathrm{~km}$} & D1 & 0.8 & 1.1 \\
\hline & D2 & 0.7 & 1.2 \\
\hline & D3 & 0.8 & 1.3 \\
\hline & D4 & 0.8 & 1.3 \\
\hline \multirow{4}{*}{$20 \mathrm{~km}$} & E1 & 1.3 & 1.5 \\
\hline & $\mathrm{E} 2$ & 1.0 & 2.3 \\
\hline & E3 & 1.5 & 1.5 \\
\hline & $\mathrm{E} 4$ & 1.3 & 2.0 \\
\hline
\end{tabular}

GPS + BeiDou signal processing in the $3^{\text {rd }}$ strategy can be seen in Table 6. This table describes that in general, the horizontal accuracies for all baselines have almost the same accuracy with the $1^{\text {st }}$ and $2^{\text {nd }}$ accuracies but $\mathrm{C} 3$ and $\mathrm{E} 3$ have accuracy more than $1.5 \mathrm{~cm}$ that is $3.3 \mathrm{~cm}$ and 1.7 $\mathrm{cm}$ for $\mathrm{C} 3$ and $\mathrm{E} 3$ respectively. The vertical accuracies for length of baselines $\leq 5 \mathrm{~km}$ has the same value with the $1^{\text {st }}$ and $2^{\text {nd }}$ strategies, however many baselines have more 2 $\mathrm{cm}$ accuracies for $10-20 \mathrm{~km}$ baselines.

Coordinate accuracy obtained from the $4^{\text {th }}$ strategy, ie GLONASS + BeiDou Satellite signal processing can be seen in the Table 7 . Horizontal accuracies for $1-5 \mathrm{~km}$ baseline length are less than $1 \mathrm{~cm}$ and vertical accuracies are less than $1.5 \mathrm{~cm}$. Generally, the horizontal accuracies for $10-20 \mathrm{~km}$ baseline are less than $20 \mathrm{~cm}$, although some points have more than $20 \mathrm{~cm}$ such as $\mathrm{C} 1(44.5 \mathrm{~cm})$ and E3 $(30.4 \mathrm{~cm})$. Vertical accuracies in these baselines almost have similar accuracy with horizontal accuracies. Some points have accuracy more than $20 \mathrm{~cm}$, namely $\mathrm{C} 1$ $(38.0 \mathrm{~cm})$, E2 $(25.4 \mathrm{~cm})$ and E3 $(28.3 \mathrm{~cm})$.
Table 5. Coordinate accuracy from GPS + GLONASS processing $\left(2^{\text {nd }}\right.$ Strategy $)$

\begin{tabular}{|c|c|r|r|}
\hline \multirow{4}{*}{ Category } & Point & $\begin{array}{c}\text { Horizontal } \\
\text { RMS (m) }\end{array}$ & $\begin{array}{c}\text { Vertical } \\
\text { RMS } \\
(\mathrm{m})\end{array}$ \\
\hline \multirow{4}{*}{$1 \mathrm{~km}$} & A1 & 0.2 & 0.5 \\
\cline { 2 - 4 } & A2 & 0.3 & 0.6 \\
\cline { 2 - 4 } & A3 & 0.5 & 0.8 \\
\cline { 2 - 4 } & A4 & 0.3 & 0.4 \\
\hline \multirow{5}{*}{$5 \mathrm{~km}$} & B1 & 0.3 & 0.5 \\
\cline { 2 - 4 } & B2 & 0.3 & 0.4 \\
\cline { 2 - 4 } & B3 & 0.4 & 0.8 \\
\cline { 2 - 4 } & B4 & 0.3 & 0.5 \\
\hline \multirow{5}{*}{$15 \mathrm{~km}$} & C1 & 1.1 & 2.0 \\
\cline { 2 - 4 } & C2 & 1.4 & 2.3 \\
\cline { 2 - 4 } & C3 & 1.9 & 1.4 \\
\cline { 2 - 4 } & C4 & 0.7 & 1.2 \\
\hline \multirow{5}{*}{$20 \mathrm{~km}$} & D1 & 0.8 & 1.1 \\
\cline { 2 - 4 } & D2 & 0.7 & 1.2 \\
\cline { 2 - 4 } & D3 & 0.8 & 1.3 \\
\cline { 2 - 4 } & D4 & 0.8 & 1.3 \\
\hline \multirow{5}{*}{} & E1 & 1.3 & 1.5 \\
\cline { 2 - 4 } & E2 & 1.0 & 2.3 \\
\cline { 2 - 4 } & E3 & 1.5 & 1.5 \\
\cline { 2 - 4 } & E4 & 1.3 & 2.0 \\
\hline
\end{tabular}

Table 8 shows that the accuracy of the $5^{\text {th }}$ strategy (only GPS processing). It can be seen that the horizontal accuracies for the baseline length less than or equal $5 \mathrm{~km}$ are $\leq 1.0 \mathrm{~cm}$ and baseline between $10 \mathrm{~km}$ and $20 \mathrm{~km}$ have accuracies less than $2.0 \mathrm{~cm}$. Except A3, the vertical accuracies for baseline $1-5 \mathrm{~km}$ are $\leq 1.0 \mathrm{~cm}$ and for baseline $10-20 \mathrm{~km}$ have accuracies less than $6.0 \mathrm{~cm}$.

Finally, the $6^{\text {th }}$ strategy used only GLONASS satellite signal describes that the horizontal accuracies for less than or equal $5 \mathrm{~km}$ baseline have $\leq 1.0 \mathrm{~cm}$ and their vertical accuracies are less than $1.5 \mathrm{~cm}$. Some points in the baseline length between 10 and $20 \mathrm{~km}$ have accuracies more than $30 \mathrm{~cm}$ both horizontally and vertically. Therefore, GLONASS data cannot be used to observe ground control point for high resolution satellite image, lidar and airborne photogrammetry. In addition, this strategy also cannot be used to observe ground control points for land surveying and mapping. 
Table 6. Coordinate accuracy from GPS + BeiDou processing ( $3^{\text {rd }}$ Strategy)

\begin{tabular}{|c|c|c|c|}
\hline Category & Point & $\begin{array}{l}\text { Horizontal } \\
\text { RMS }(\mathrm{cm})\end{array}$ & $\begin{array}{c}\text { Vertical } \\
\text { RMS (cm) }\end{array}$ \\
\hline \multirow{4}{*}{$1 \mathrm{~km}$} & A1 & 0.2 & 0.4 \\
\hline & $\mathrm{A} 2$ & 0.4 & 0.7 \\
\hline & A3 & 1.0 & 1.9 \\
\hline & A4 & 0.5 & 0.9 \\
\hline \multirow{4}{*}{$5 \mathrm{~km}$} & B1 & 0.3 & 0.5 \\
\hline & B2 & 0.4 & 0.5 \\
\hline & B3 & 0.5 & 1.0 \\
\hline & B4 & 0.3 & 0.5 \\
\hline \multirow{4}{*}{$10 \mathrm{~km}$} & $\mathrm{C} 1$ & 1.0 & 2.4 \\
\hline & $\mathrm{C} 2$ & 1.5 & 2.3 \\
\hline & $\mathrm{C} 3$ & 3.3 & 5.3 \\
\hline & $\mathrm{C} 4$ & 0.6 & 1.1 \\
\hline \multirow{4}{*}{$15 \mathrm{~km}$} & D1 & 0.8 & 1.2 \\
\hline & D2 & 0.8 & 1.6 \\
\hline & D3 & 0.9 & 1.3 \\
\hline & D4 & 0.9 & 1.9 \\
\hline \multirow{4}{*}{$20 \mathrm{~km}$} & E1 & 1.3 & 1.6 \\
\hline & E2 & 1.1 & 2.8 \\
\hline & E3 & 1.7 & 2.7 \\
\hline & E4 & 1.4 & 1.8 \\
\hline
\end{tabular}

Table 7. Coordinate accuracy from GLONASS + BeiDou processing ( $4^{\text {th }}$ Strategy)

\begin{tabular}{|c|c|c|c|}
\hline Category & Point & $\begin{array}{l}\text { Horizontal } \\
\text { RMS (cm) }\end{array}$ & $\begin{array}{c}\text { Vertical } \\
\text { RMS }(\mathrm{cm})\end{array}$ \\
\hline \multirow{4}{*}{$1 \mathrm{~km}$} & A1 & 1.0 & 1.4 \\
\hline & $\mathrm{A} 2$ & 0.5 & 1.0 \\
\hline & A3 & 0.6 & 0.8 \\
\hline & A4 & 0.4 & 0.4 \\
\hline \multirow{4}{*}{$5 \mathrm{~km}$} & B1 & 0.6 & 1.0 \\
\hline & B2 & 0.5 & 0.5 \\
\hline & B3 & 0.5 & 1.0 \\
\hline & B4 & 0.7 & 1.2 \\
\hline \multirow{4}{*}{$10 \mathrm{~km}$} & $\mathrm{C} 1$ & 44.5 & 38.0 \\
\hline & $\mathrm{C} 2$ & 8.8 & 17.8 \\
\hline & C3 & 1.6 & 1.1 \\
\hline & $\mathrm{C} 4$ & 1.8 & 4.6 \\
\hline \multirow{4}{*}{$15 \mathrm{~km}$} & D1 & 1.3 & 1.8 \\
\hline & D2 & 1.7 & 1.9 \\
\hline & D3 & 1.3 & 2.1 \\
\hline & D4 & 5.1 & 6.4 \\
\hline Category & Point & $\begin{array}{l}\text { Horizontal } \\
\text { RMS }(\mathrm{cm})\end{array}$ & $\begin{array}{c}\text { Vertical } \\
\text { RMS (cm) }\end{array}$ \\
\hline
\end{tabular}

\begin{tabular}{|c|c|c|c|}
\hline \multirow{3}{*}{$20 \mathrm{~km}$} & E1 & 5.6 & 11.3 \\
\cline { 2 - 4 } & E2 & 17.8 & 25.4 \\
\cline { 2 - 4 } & E3 & 30.4 & 28.3 \\
\cline { 2 - 4 } & E4 & 2.2 & 2.5 \\
\hline
\end{tabular}

Table 8. Coordinate accuracy from only GPS processing ( $5^{\text {th }}$ Strategy)

\begin{tabular}{|c|c|c|c|}
\hline Category & Point & $\begin{array}{l}\text { Horizontal } \\
\text { RMS }(\mathrm{cm})\end{array}$ & $\begin{array}{c}\text { Vertical } \\
\text { RMS (cm) }\end{array}$ \\
\hline \multirow{4}{*}{$1 \mathrm{~km}$} & A1 & 2.0 & 4.0 \\
\hline & $\mathrm{A} 2$ & 4.0 & 7.0 \\
\hline & A3 & 1.0 & 1.9 \\
\hline & A4 & 0.5 & 0.9 \\
\hline \multirow{4}{*}{$5 \mathrm{~km}$} & B1 & 0.3 & 0.5 \\
\hline & B2 & 0.4 & 0.5 \\
\hline & B3 & 0.5 & 1.0 \\
\hline & B4 & 0.3 & 0.5 \\
\hline \multirow{4}{*}{$10 \mathrm{~km}$} & $\mathrm{C} 1$ & 1.0 & 2.4 \\
\hline & $\mathrm{C} 2$ & 1.5 & 2.3 \\
\hline & $\mathrm{C} 3$ & 3.3 & 5.3 \\
\hline & $\mathrm{C} 4$ & 0.6 & 1.1 \\
\hline \multirow{4}{*}{$15 \mathrm{~km}$} & D1 & 0.8 & 1.2 \\
\hline & D2 & 0.8 & 1.2 \\
\hline & D3 & 0.9 & 1.3 \\
\hline & D4 & 0.9 & 1.9 \\
\hline \multirow{4}{*}{$20 \mathrm{~km}$} & E1 & 1.3 & 1.6 \\
\hline & E2 & 1.1 & 2.8 \\
\hline & E3 & 1.7 & 2.7 \\
\hline & $\mathrm{E} 4$ & 1.4 & 1.8 \\
\hline
\end{tabular}

Table 9. Coordinate accuracy from only GLONASS processing ( $6^{\text {th }}$ Strategy)

\begin{tabular}{|c|c|r|r|}
\hline Category & Point & $\begin{array}{r}\text { Horizontal } \\
\text { RMS }(\mathrm{cm})\end{array}$ & $\begin{array}{c}\text { Vertical } \\
\text { RMS (cm) }\end{array}$ \\
\hline \multirow{4}{*}{$1 \mathrm{~km}$} & A1 & 1.0 & 1.4 \\
\cline { 2 - 4 } & A2 & 0.5 & 1.0 \\
\cline { 2 - 4 } & A3 & 0.6 & 0.8 \\
\cline { 2 - 4 } & A4 & 0.4 & 0.4 \\
\hline \multirow{5}{*}{$5 \mathrm{~km}$} & B1 & 0.6 & 1.0 \\
\cline { 2 - 4 } & B2 & 0.5 & 1.0 \\
\cline { 2 - 4 } & B3 & 0.5 & 1.0 \\
\cline { 2 - 4 } & B4 & 0.7 & 1.2 \\
\hline \multirow{4}{*}{$10 \mathrm{~km}$} & $\mathrm{C} 1$ & 36.3 & 62.4 \\
\cline { 2 - 4 } & $\mathrm{C} 2$ & 58.5 & 35.9 \\
\cline { 2 - 4 } & $\mathrm{C} 3$ & 1.6 & 1.1 \\
\cline { 2 - 4 } & $\mathrm{C} 4$ & 1.8 & 4.5 \\
\hline
\end{tabular}




\begin{tabular}{|c|c|r|r|}
\hline \multirow{4}{*}{$15 \mathrm{~km}$} & D1 & 1.3 & 1.8 \\
\cline { 2 - 4 } & D2 & 1.8 & 1.9 \\
\cline { 2 - 4 } & D3 & 1.3 & 2.1 \\
\cline { 2 - 4 } & D4 & 5.6 & 6.8 \\
\hline \multirow{4}{*}{$20 \mathrm{~km}$} & E1 & 5.3 & 11.1 \\
\cline { 2 - 4 } & E2 & 1.8 & 25.4 \\
\cline { 2 - 4 } & E3 & 33.8 & 30.1 \\
\cline { 2 - 4 } & E4 & 2.2 & 2.5 \\
\hline
\end{tabular}

\subsection{Coordinate accuracy analysis of baseline processing}

The coordinates accuracies of the data processing with the six strategies mentioned above can be categorized based on the length of the baseline. The accuracy of coordinates of each category from A (1 km) to E $(20 \mathrm{~km})$ can be seen in Tables 10 to Table 14.

Table 10 shows that all strategies resulted horizontal accuracy for category A $(1 \mathrm{~km}$ baseline $) \leq 1 \mathrm{~cm}$. However only the $1^{\text {st }}$ strategy and the $2^{\text {nd }}$ strategy produced vertical accuracies less than or equal $1 \mathrm{~cm}$ and other strategies have accuracy almost $2 \mathrm{~cm}$ for some points.

Table 10. Coordinate accuracy category A (length of baseline $1 \mathrm{~km}$ )

\begin{tabular}{|c|l|c|c|c|c|}
\hline Strategy & Accuracy & $\begin{array}{c}\text { A1 } \\
(\mathrm{cm})\end{array}$ & $\begin{array}{c}\mathrm{A} 2 \\
(\mathrm{~cm})\end{array}$ & $\begin{array}{c}\mathrm{A} 3 \\
(\mathrm{~cm})\end{array}$ & $\begin{array}{c}\mathrm{A} 4 \\
(\mathrm{~cm})\end{array}$ \\
\hline \multirow{2}{*}{$1^{\text {st }}$} & Horizontal & 0.2 & 0.3 & 0.5 & 0.3 \\
\cline { 2 - 6 } & Vertical & 0.5 & 0.6 & 0.8 & 0.4 \\
\hline \multirow{2}{*}{$2^{\text {nd }}$} & Horizontal & 0.2 & 0.3 & 0.5 & 0.3 \\
\cline { 2 - 6 } & Vertical & 0.5 & 0.6 & 0.8 & 0.4 \\
\hline \multirow{2}{*}{$3^{\text {rd }}$} & Horizontal & 0.2 & 0.4 & 1.0 & 0.5 \\
\cline { 2 - 6 } & Vertical & 0.4 & 0.7 & 1.9 & 0.9 \\
\hline \multirow{2}{*}{$4^{\text {th }}$} & Horizontal & 1.0 & 0.5 & 0.6 & 0.4 \\
\cline { 2 - 6 } & Vertical & 1.4 & 1.0 & 0.8 & 0.4 \\
\hline \multirow{2}{*}{$5^{\text {th }}$} & Horizontal & 0.2 & 0.4 & 1.0 & 0.5 \\
\cline { 2 - 6 } & Vertical & 0.4 & 0.7 & 1.9 & 0.9 \\
\hline \multirow{2}{*}{$6^{\text {th }}$} & Horizontal & 1.0 & 0.5 & 0.6 & 0.4 \\
\cline { 2 - 6 } & Vertical & 1.4 & 1.0 & 0.8 & 0.4 \\
\hline
\end{tabular}

All coordinate accuracies for category B ( $5 \mathrm{~km}$ baseline) can be seen on Table 11. In this case all points have horizontal and vertical accuracies less than $1.25 \mathrm{~cm}$. This category has better accuracy than category A.
Table 11. Coordinate accuracy category B (length of baseline $5 \mathrm{~km}$ )

\begin{tabular}{|c|l|c|c|c|c|}
\hline Strategy & Accuracy & $\begin{array}{c}\text { B1 } \\
(\mathrm{cm})\end{array}$ & $\begin{array}{c}\mathrm{B} 2 \\
(\mathrm{~cm})\end{array}$ & $\begin{array}{c}\mathrm{B} 3 \\
(\mathrm{~cm})\end{array}$ & $\begin{array}{c}\mathrm{B} 4 \\
(\mathrm{~cm})\end{array}$ \\
\hline \multirow{2}{*}{$1^{\text {st }}$} & Horizontal & 0.3 & 0.3 & 0.4 & 0.3 \\
\cline { 2 - 6 } & Vertical & 0.5 & 0.4 & 0.8 & 0.5 \\
\hline \multirow{2}{*}{$2^{\text {nd }}$} & Horizontal & 0.3 & 0.3 & 0.4 & 0.3 \\
\cline { 2 - 6 } & Vertical & 0.5 & 0.4 & 0.8 & 0.5 \\
\hline \multirow{2}{*}{$3^{\text {rd }}$} & Horizontal & 0.3 & 0.4 & 0.5 & 0.3 \\
\cline { 2 - 6 } & Vertical & 0.5 & 0.5 & 1.0 & 0.5 \\
\hline \multirow{2}{*}{$4^{\text {th }}$} & Horizontal & 0.6 & 0.5 & 0.5 & 0.7 \\
\cline { 2 - 6 } & Vertical & 1.0 & 0.5 & 1.0 & 1.2 \\
\hline \multirow{2}{*}{$5^{\text {th }}$} & Horizontal & 0.3 & 0.4 & 0.5 & 0.3 \\
\cline { 2 - 6 } & Vertical & 0.5 & 0.5 & 1.0 & 0.5 \\
\hline \multirow{2}{*}{$6^{\text {th }}$} & Horizontal & 0.6 & 0.5 & 0.5 & 0.7 \\
\cline { 2 - 6 } & Vertical & 1.0 & 1.0 & 1.0 & 1.2 \\
\hline
\end{tabular}

Horizontal accuracies for the $10 \mathrm{~km}$ length of baseline can be seen Table 12. This table shows that horizontal accuracies are less than $2 \mathrm{~cm}$ for the $1^{\text {st }}$ and $2^{\text {nd }}$ strategies, otherwise generally other strategies have more than $2 \mathrm{~cm}$ accuracies. Many points have more than $35 \mathrm{~cm}$ accuracies such as $\mathrm{C} 1$ for $4^{\text {th }}$ and $6^{\text {th }}$ strategies and $\mathrm{C} 2$ for $6^{\text {th }}$ strategy. The $1^{\text {st }}$ and the $2^{\text {nd }}$ strategies have vertical accuracies less than $2.5 \mathrm{~cm}$ and the $4^{\text {th }}$ and $6^{\text {th }}$ strategies have $38 \mathrm{~cm}$ and $62.4 \mathrm{~cm}$ for $\mathrm{C} 1$ and $17.8 \mathrm{~cm}$ and $35.9 \mathrm{~cm}$ for $\mathrm{C} 2$.

Table 12. Coordinate accuracy category $\mathrm{C}$ (length of baseline $10 \mathrm{~km}$ )

\begin{tabular}{|c|l|c|c|c|c|}
\hline Strategy & Accuracy & $\begin{array}{c}\mathrm{C} 1 \\
(\mathrm{~cm})\end{array}$ & $\begin{array}{c}\mathrm{C} 2 \\
(\mathrm{~cm})\end{array}$ & $\begin{array}{c}\mathrm{C} 3 \\
(\mathrm{~cm})\end{array}$ & $\begin{array}{c}\mathrm{C} 4 \\
(\mathrm{~cm})\end{array}$ \\
\hline \multirow{2}{*}{$1^{\text {st }}$} & Horizontal & 1.1 & 1.4 & 1.9 & 0.7 \\
\cline { 2 - 6 } & Vertical & 2.0 & 2.3 & 1.4 & 1.2 \\
\hline \multirow{2}{*}{$2^{\text {nd }}$} & Horizontal & 1.1 & 1.4 & 1.9 & 0.7 \\
\cline { 2 - 6 } & Vertical & 2.0 & 2.3 & 1.4 & 1.2 \\
\hline \multirow{2}{*}{$3^{\text {rd }}$} & Horizontal & 1.0 & 1.5 & 3.3 & 0.6 \\
\cline { 2 - 6 } & Vertical & 2.4 & 2.3 & 5.3 & 1.1 \\
\hline \multirow{2}{*}{$4^{\text {th }}$} & Horizontal & 44.5 & 8.8 & 1.6 & 1.8 \\
\cline { 2 - 6 } & Vertical & 38.0 & 17.8 & 1.1 & 4.6 \\
\hline \multirow{2}{*}{$5^{\text {th }}$} & Horizontal & 1.0 & 1.5 & 3.3 & 0.6 \\
\cline { 2 - 6 } & Vertical & 2.4 & 2.3 & 5.3 & 1.1 \\
\hline \multirow{2}{*}{$6^{\text {th }}$} & Horizontal & 36.3 & 58.5 & 1.6 & 1.8 \\
\cline { 2 - 6 } & Vertical & 62.4 & 35.9 & 1.1 & 4.5 \\
\hline
\end{tabular}

In general, horizontal and vertical accuracies for category D have better than category C. All accuracies in category D (Table 13) are less than or equal $2.1 \mathrm{~cm}$. Some points have accuracies more than $5 \mathrm{~cm}$ such as D4 for $4^{\text {th }}$ and $6^{\text {th }}$ strategies. 
Table 13. Coordinate accuracy category D (length of baseline $15 \mathrm{~km}$ )

\begin{tabular}{|c|l|c|c|c|c|}
\hline Strategy & Accuracy & $\begin{array}{c}\text { D1 } \\
(\mathrm{cm})\end{array}$ & $\begin{array}{c}\mathrm{D} 2 \\
(\mathrm{~cm})\end{array}$ & $\begin{array}{c}\mathrm{D} 3 \\
(\mathrm{~cm})\end{array}$ & $\begin{array}{c}\mathrm{D} 4 \\
(\mathrm{~cm})\end{array}$ \\
\hline \multirow{2}{*}{$1^{\text {st }}$} & Horizontal & 0.8 & 0.7 & 0.8 & 0.8 \\
\cline { 2 - 6 } & Vertical & 1.1 & 1.2 & 1.3 & 1.3 \\
\hline \multirow{2}{*}{$2^{\text {nd }}$} & Horizontal & 0.8 & 0.7 & 0.8 & 0.8 \\
\cline { 2 - 6 } & Vertical & 1.1 & 1.2 & 1.3 & 1.3 \\
\hline \multirow{2}{*}{$3^{\text {rd }}$} & Horizontal & 0.8 & 0.8 & 0.9 & 0.9 \\
\cline { 2 - 6 } & Vertical & 1.2 & 1.6 & 1.3 & 1.9 \\
\hline \multirow{2}{*}{$4^{\text {th }}$} & Horizontal & 1.3 & 1.7 & 1.3 & 5.1 \\
\cline { 2 - 6 } & Vertical & 1.8 & 1.9 & 2.1 & 6.4 \\
\hline \multirow{2}{*}{$5^{\text {th }}$} & Horizontal & 0.8 & 0.8 & 0.9 & 0.9 \\
\cline { 2 - 6 } & Vertical & 1.2 & 1.2 & 1.3 & 1.9 \\
\hline \multirow{2}{*}{$6^{\text {th }}$} & Horizontal & 1.3 & 1.8 & 1.3 & 5.6 \\
\cline { 2 - 6 } & Vertical & 1.8 & 1.9 & 2.1 & 6.8 \\
\hline
\end{tabular}

Finally, coordinate accuracies for category E can be seen in Table E. This table shows that horizontal and vertical accuracies are less than $3 \mathrm{~cm}$ for $1^{\text {st }}, 2^{\text {nd }}, 3^{\text {rd }}$ and $4^{\text {th }}$ strategies, however in the $4^{\text {th }}$ and $6^{\text {th }}$ strategies almost all points have accuracies more than $11 \mathrm{~cm}$ except all point in $\mathrm{E} 4$.

Table 14. Coordinate accuracy category E (length of baseline $20 \mathrm{~km}$ )

\begin{tabular}{|c|l|c|c|c|c|}
\hline Strategy & Accuracy & $\begin{array}{c}\text { E1 } \\
(\mathrm{cm})\end{array}$ & $\begin{array}{c}\text { E2 } \\
(\mathrm{cm})\end{array}$ & $\begin{array}{c}\text { E3 } \\
(\mathrm{cm})\end{array}$ & $\begin{array}{c}\text { E4 } \\
(\mathrm{cm})\end{array}$ \\
\hline \multirow{2}{*}{$1^{\text {st }}$} & Horizontal & 1.3 & 1.0 & 1.5 & 1.3 \\
\cline { 2 - 6 } & Vertical & 1.5 & 2.3 & 1.5 & 2.0 \\
\hline \multirow{2}{*}{$2^{\text {nd }}$} & Horizontal & 1.3 & 1.0 & 1.5 & 1.3 \\
\cline { 2 - 6 } & Vertical & 1.5 & 2.3 & 1.5 & 2.0 \\
\hline \multirow{2}{*}{$3^{\text {rd }}$} & Horizontal & 1.3 & 1.1 & 1.7 & 1.4 \\
\cline { 2 - 6 } & Vertical & 1.6 & 2.8 & 2.7 & 1.8 \\
\hline \multirow{2}{*}{$4^{\text {th }}$} & Horizontal & 5.6 & 17.8 & 30.4 & 2.2 \\
\cline { 2 - 6 } & Vertical & 11.3 & 25.4 & 28.3 & 2.5 \\
\hline \multirow{2}{*}{$5^{\text {th }}$} & Horizontal & 1.3 & 1.1 & 1.7 & 1.4 \\
\cline { 2 - 6 } & Vertical & 1.6 & 2.8 & 2.7 & 1.8 \\
\hline \multirow{2}{*}{$6^{\text {th }}$} & Horizontal & 5.3 & 18.0 & 33.8 & 2.2 \\
\cline { 2 - 6 } & Vertical & 11.1 & 25.4 & 30.1 & 2.5 \\
\hline
\end{tabular}

\section{Conclusion}

Based on the results of this research, it can be concluded that:

1. The $1^{\text {st }}($ GPS + GLONASS + BeiDou $)$ and $2^{\text {nd }}$ (GPS + GLONASS) strategy have the same horizontal and vertical accuracies for all categories $(1-20 \mathrm{~km})$ whereas horizontal accuracies are less than $2 \mathrm{~cm}$ and vertical are accuracies less than $2.5 \mathrm{~cm}$.
2. All strategies have horizontal accuracies less than or equal $1 \mathrm{~cm}$ and vertical accuracies less than $2 \mathrm{~cm}$ for baseline $\leq 5 \mathrm{~km}$.

\section{Acknowledgment}

We wish to acknowledge PT. Geosolution Pratama Nusantara for the GNSS HiTarget V30 equipment thus we can complete this research

\section{References}

1. H.C. Chen, Y.S. Huang, K.W Chiang, M Yang, And R.J. Rauthe. Performance Comparison Between GPS And Beidou-2/Compass: A Perspective from Asia. Journal of The Chinese Institute of Engineers, Vol. 32, No. 5, Pp. 679689. 2009.

2. Novatel. An Introduction to GNSS GPS, GLONASS, BeiDou, Galileo and other Global Navigation Satellite Systems. Novatel Inc. Second Edition 2015.

3. C.D. Ghilani and P.R. Wolf. Elementary Surveying. An introduction to Geomatics. $13^{\text {th }}$ edition. Prentice Hall. 2012

4. G. Seeber. Satellite Geodesy 2nd Edition. Walter de Gruyter · Berlin 2003

5. H.Z. Abidin, A. Jones, dna J. Kahar. Survei Dengan GPS. Cetakan Ketiga. Jakarta: PT Pradnya Paramita. 2011.

6. B. Rudianto., Y. Izman., Analisis Komparatif Ketelitian Posisi Titik Hasil Pengukuran dari Satelit GPS dan Satelit GLONASS. Institut Teknologi Nasional. 2011.

7. I. Gumilar , A.I. Pamungkas, H.Z. Abidin, B. Bramanto, F.S. Adi. 2016. The Contribution of BeiDou Positioning System for Accuracy Improvement : A Perspective from Bandung, Indonesia. International Symposium on GNSS at Tainan, Taiwan. 2016.

8. Hi-Target. V30/50 GNSS RTK System Manual. Hi-Target. Surveying Instrument Co., Ltd. 2010 